\title{
Strongly-Coupled Direct Numerical Simulation of Thermal Turbulence in Turbine-Blade Cooling Channel with Rib-Tabulator and Surface- Roughness Structures
}

\author{
Ting Y $\mathbf{u}^{1}$, Yinqing $\mathrm{Zu}^{1}$, Hongyi $\mathrm{Xu}^{1,2}$ \\ ${ }^{1}$ Aeronautics and Astronautics Department, Fudan University \\ Shanghai China, 200433 \\ ${ }^{2}$ Department of Engineering Mechanics, State Key Laboratory of Structure Analysis for Industrial Equipment \\ International Research Centre for Computational Mechanics, Dalian University of Technology \\ Dalian China, 116024 \\ hongyi_xu@fudan.edu.cn
}

\begin{abstract}
The paper applied the state-of-the-art flow simulation method, i.e. the Direct Numerical Simulation (DNS), and strongly coupled the DNS with the heat-transfer governing equation to solve the thermal-turbulence problem in a turbine-blade cooling channel with rib-tabulator structures. In order to capture the thermal-fluid phenomena and to more accurately simulate the flow and temperature fields in reality, two innovative approaches were applied to the simulations. First, the surface roughness effects of the cooling vane was considered by including the roughness geometry in the DNS and an innovative immersed-boundary method were invented to handle the geometry complexities due to the roughness. Secondly, the time-sequencing fully-developed turbulent inflow conditions were generated through the temporal DNS of turbulence in a channel and these conditions guaranteed the qualities of the spatial DNS simulation of cooling vane with ribs, which were expected to provide a variety of advantages over the conventional Reynolds-averaged Navier-Stokes (RANS) approach. The preliminary results presented the typical wall-turbulence characteristics, such as the near-wall coherent structures for a regular smoothed wall and more interesting flow structures caused by a wall roughness. The coupled heattransfer simulation captured the temperature and its derivative fields, which exhibited the attractive coherent streaky patterns associated with the turbulence.
\end{abstract}

Keywords: Direct Numerical Simulation, Heat Transfer, Strongly-coupled Simulation, Rib Tabulator

\section{Introduction}

The advanced blade-cooling technologies is one of the focal points in nowadays aero-engine research and development (R\&D) activities [1] and the target is to seek more effective cooling methods which make the blade capable of withstanding higher gas temperature and therefore, achieving higher thrust ratio with more operational reliability. Within this aspect, the ever complicated cooling structures inside and outside turbine blade, such as film cooling, single and double-layer cooling vanes, rib tabulators [2], impingement holes and pin-fins[3] structures as well as their combinations tend to be more and more popular to enhance the heat transfer efficiency. The current paper applied the stateof-the-art research in fluid mechanics, namely Direct Numerical Simulation (DNS), to the representative cooling structures, such as rib tabulator, in blade cooling channels. The DNS were strongly coupled with the heat-transfer simulation to obtain the complete thermal turbulence information inside an internal cooling vane of turbine blade. To be able to capture the thermal turbulence phenomena and to more accurately mimic the flow and heat transfer inside the turbine blade, two innovative approaches were invented and were applied to the simulations in the current research. First, the surface roughness of the cooling channel was included and resolved explicitly in the simulations. An immersed boundary (IB) method coupled with the adaptive mesh refinement technology was implemented to tackle the extremely complex geometries of the surface roughness [4]. Secondly, the time-sequencing fully-developed turbulent boundary conditions were generated through the temporal DNS simulations in a channel [5] and these conditions guaranteed the qualities of the spatial DNS simulation of the cooling vane with rib tabulator structures. Based on these technologies, a large-scale parallel-computing platform were developed with the strongly-coupled DNS simulation capability of turbulent flow and heat transfer. The turbine blade cooling structures with the rib-tabulators were systematically investigated under a 
variety of conditions and configurations in industrial applications. These DNS simulation results will be collected to build a large database which contains a wealth of turbulent-thermal flow information, such as the turbulent-thermal instantaneous fields, the statistics of mean thermal flow fields, the Reynolds stresses and thermal stresses etc. The data will significantly help pushing the nowadays frontiers in each of these relevant science fields, such as the fluid mechanics, heat transfer as well as computations. Also, the database will help transforming the design and manufacture of the turbine blade from the current one based on engineering experiments and empirical data to the future one intelligently-guided by numerical simulation databank.

\section{Mathematical-Physical Models and Computational Methods}

The section provides the mathematical models with their scopes of physical application in developing the stronglycoupled direct numerical simulation capability to predict the thermal turbulence phenomena in blade-cooling structures with rib tabulator, as seen in Fig. 1.

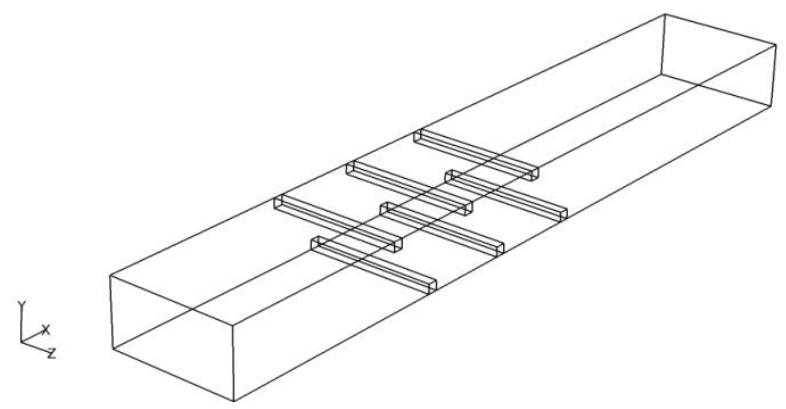

Fig. 1: Schematic drawing of cooling vane with rib-tabulator structures.

\subsection{Governing Equations}

The governing equations in the current research included the (1) mass, (2) momentum and (3) energy conservations for the unsteady flow of incompressible ideal gas in the cooling vane as shown in Fig. 1, which can be written in the nondimensional form under Cartesian coordinators as:

$$
\begin{gathered}
\frac{\partial \bar{u}_{J}}{\partial \bar{x}_{J}}=0 \\
\frac{\partial \bar{u}_{l}}{\partial \bar{t}}+\frac{\partial \bar{u}_{\imath} \bar{u}_{J}}{\partial \bar{x}_{J}}=-\frac{\partial \bar{p}}{\partial \bar{x}_{l}}+\frac{1}{R e} \frac{\partial}{\partial \bar{x}_{J}}\left(\frac{\partial \bar{u}_{l}}{\partial \bar{x}_{J}}+\frac{\partial \bar{u}_{J}}{\partial \bar{x}_{l}}\right) \\
\frac{\partial \Theta}{\partial \bar{t}}+\frac{\partial \bar{u}_{J} \Theta}{\partial \bar{x}_{J}}=\frac{1}{\operatorname{RePr}} \frac{\partial^{2} \Theta}{\partial \bar{x}_{J} \partial \bar{x}_{J}}+\frac{E c}{R e} \boldsymbol{\Phi} ; \quad \boldsymbol{\Phi}=\frac{1}{2}\left(\frac{\partial \bar{u}_{l}}{\partial \bar{x}_{J}}+\frac{\partial \bar{u}_{J}}{\partial \bar{x}_{l}}\right)\left(\frac{\partial \bar{u}_{l}}{\partial \bar{x}_{J}}+\frac{\partial \bar{u}_{J}}{\partial \bar{x}_{l}}\right)
\end{gathered}
$$

where: the subscripts $i, j=1,2,3$ represent the spatial direction of $x, y, z$, respectively, with $x$ being the streamwise and $y, z$ being the cross-streamwise directions; the repeated subscriptions imply the Einstein summation and $\Phi$ is the dissipation function; $\overline{u_{\imath}}$ and $\Theta$ are the non-dimensional velocities and the temperature with the velocity scale being the incoming average velocity and the temperature scale being the difference between the wall and incoming-stream temperatures.

The working temperature in a typical blade-cooling structures is usually around 1000k and the flow speed inside the vane is in the range of $50-100 \mathrm{~m} / \mathrm{s}$, which results in a Mach number in between $M \approx 0.08 \square 0.16$. As seen clearly, the higher sonic speed due to the high temperature makes the flow inside the cooling vane falls into the incompressible regime that validates the incompressible model assumption.

\subsection{Computation Schemes}


The second-order finite volume schemes were applied in the spatial discretizations. In temporal direction, the convection and diffusion terms were handled by the second-order time-marching schemes of Adams-Bashforth and Adams-Moulton, respectively. The velocity and pressure fields were decoupled by the fractional step method [6]. To achieve a high-performance solution efficiency, the robust Flexible-Cycle Additive-Correction (FCAC)-multigrid (MG) in [7] was coupled with the IB methods to tackle the unsteady multi-scale turbulence motions under the conditions of complex surface-roughness geometries.

\subsection{Surface Roughness Geometry}

Since the high-temperature blade cooling vane of modern aero-engine are usually manufactured at the scale of several millimeters and the surface roughness are usually at the scales in between several microns to tens of microns, the effects of the roughness is no longer negligible and has to be considered and be resolved in the DNS. On the other hand, the computational grids have to be sufficiently refined to directly resolve the flow-structures near the surface roughness. The $\mathrm{X}$-ray scanning technologies were applied to obtain the geometric contours of the roughness on the cooling-vane surface and the computational grids near the roughened surfaces were generated using the IB method. Fig. 2 demonstrated the roughness distributions on the cooling channel surface and the computational grids generated near the surfaces with the rib-tabulator structures.
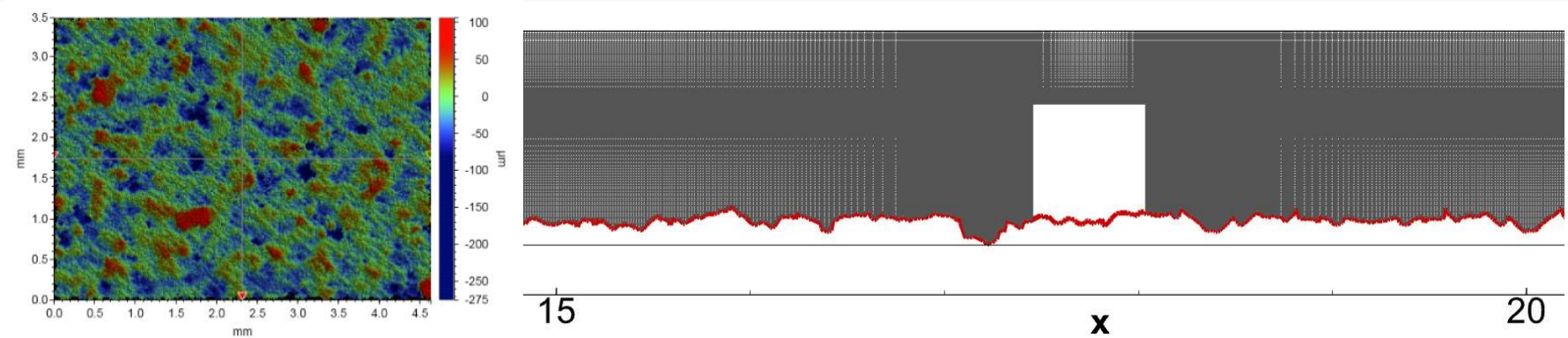

Fig. 2: Typical roughness structures on the cooling vane surface with rib-tabulator and the computational grid generated using IB method.

\subsection{Time-Sequencing Turbulent Inflow Conditions}

To accurately simulation the turbulence inside the cooling channel and to capture the heat transfer under turbulent flow conditions, the realistic turbulent inflow conditions at the inlet is a critically important issue in the DNS. As suggested [5], the current research generated the time-sequencing fully-developed turbulent inflow conditions through a separate temporal DNS in a channel with an equivalent Reynolds number to the flow in cooling vane. These conditions guaranteed the qualities of the spatial DNS of the cooling vane with rib tabulators, as seen in Fig.3.

\section{Results and Discussions}

The DNS simulations were preliminarily conducted and conceptually implemented for the two-dimensional cooling channel with rib-tabulator arranged in a staggered configuration between the upper and lower surfaces, as seen in Fig. 4. The Reynolds number was set at $\mathrm{Re}=10,000$, which satisfied most of the industrial applications. The channel surfaces roughness was selected at $\mathrm{Ra}=1.6$, which was obtained by X-ray scanning the metal surface shown in Fig. 2. The simulations were performed for the channel with both smooth and rough surfaces under a uniform and a fully-developed turbulent inflow conditions. The non-dimensional wall and rib temperatures were set at 1.0 as the boundary conditions for Eq. (3).

Fig. 4(a) demonstrated the instantaneous temperature distributions as the strongly-coupled flow and temperature fields developing in the cooling vane with smoothed surface and staggered-rib arrangement. Fig. 4(b) exhibited its counterpart with roughened surface. Although the flows was still not fully developed at the time of paper writing, the striking features of the strongly-coupled flow and thermal interaction phenomena were seen in the cooling channels with rib structures, including the thermal boundary layers development in the front of leading ribs(R1), the typical backward-facing step thermal flow patterns near the leeward surface of the ribs(R2) and the flow-heat convections along the horizontal surfaces at the top of the ribs(R3). The comparison between Fig. 4(a) and (b) indicated that the wall-roughness significantly affected 
the flow and heat transfer, particularly in the near wall regions. Fig. 5(a) and (b) presented the temperature gradient component of $\partial \Theta / \partial y$ which physically represented the heat conduction intensity in y direction. Fig. 5(a) and (b) exhibited characteristic and striking streaky structures in a variety of zones, such as R1, R2 and R3, and these structures were very similar to the well-known coherent structures of turbulence, indicating that turbulence had strong effects in the formations of these heattransfer structures and patterns. Statistical analyses of these structures will result in important findings of heat transfer mechanisms affected by the turbulence in a ribbed channel.

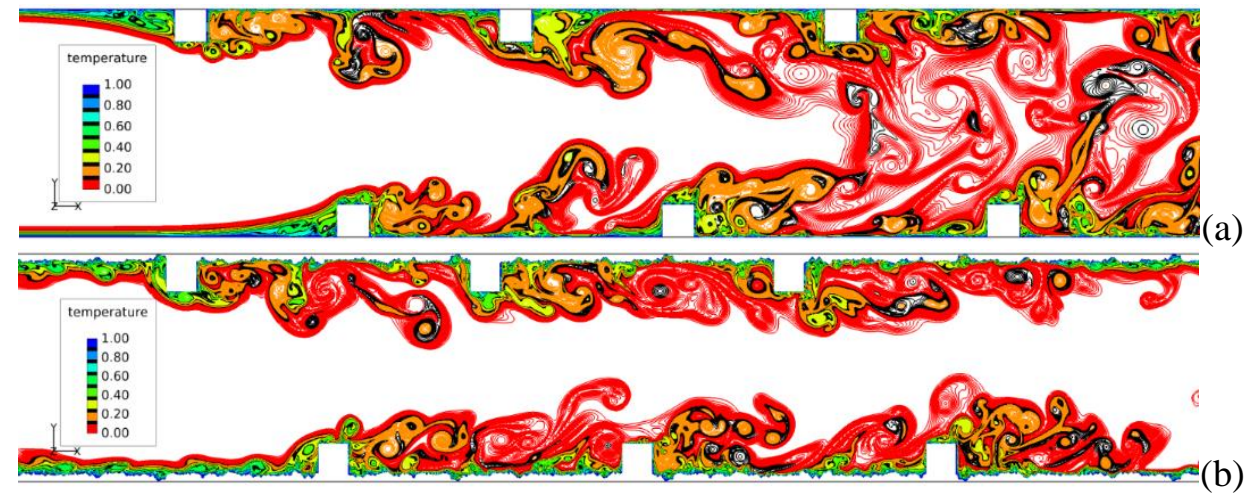

Fig. 4: Instantaneous temperature fields in the ribbed channel with (a) smoothed surface; (b) roughened surface.
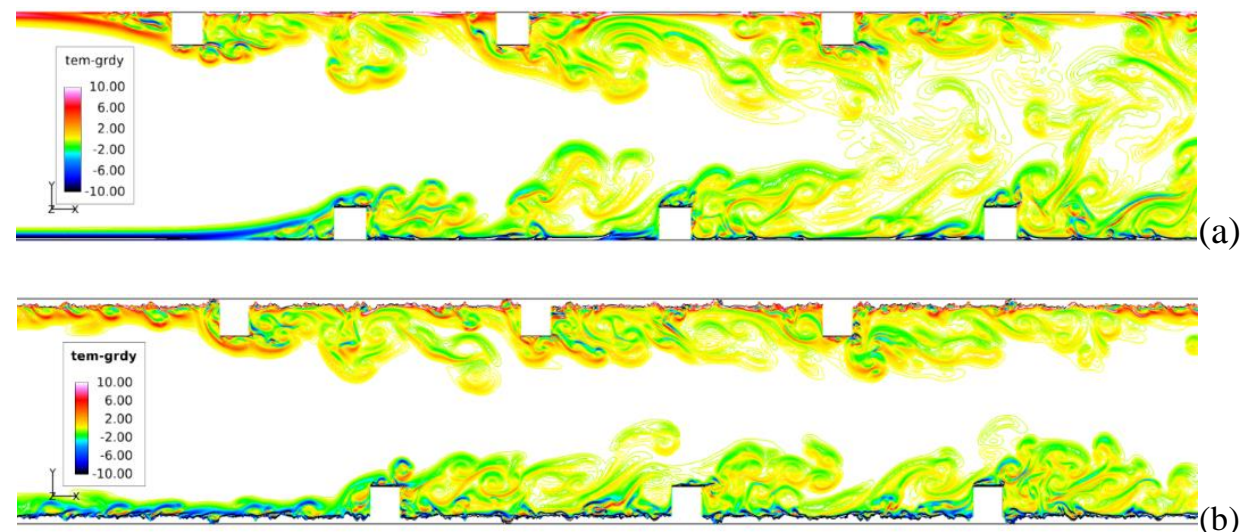

Fig. 5: Instantaneous temperature gradient of $\partial \Theta / \partial \mathrm{y}$ in the ribbed channel with (a) smoothed and (b) roughened surface.

\section{Conclusion}

Strongly-coupled DNS were conceptually designed, implemented and conducted in two-dimensional ribbed channels configurations with smoothed and roughened surfaces. The time-sequencing fully-developed turbulent inflow conditions were imposed at the inlet of the channel. The wall roughness were found significantly affecting the heat transfer process in the ribbed channel. The temperature gradient fields were discovered bearing similar streaky characteristics comparing to the well-known turbulence coherent structures. The current work laid out a solid foundation, based on which a large-scale three-dimensional DNS will be carried out in the near future.

\section{Acknowledgements}

The authors would like to acknowledge the financial support from the Natural Science Foundation of China (NSFC), Major Research Plan under the Grant No. 91434112 and from the United Innovation Program of Shanghai Commercial Aircraft Engine with the Grant No. AR908.D1RW.002 (the program was founded by Shanghai Municipal Commission of Economy and Information, Shanghai Municipal Education Commission and AECC Commercial Aircraft Engine Co., LTD). The research was initiated by the funds from Fudan University under the Shanghai Thousand Talents Program.

\section{References}


[1] J. C. Han, "Recent Studies in Turbine Blade Cooling," International Journal of Rotating Machinery, vol. 10, no. 6, pp. 443-457, 2004.

[2] J. C. Han, Y. M. Zhang, C. P. Lee, "Augmented heat transfer in square channels with parallel, crossed, and V-shaped angled Ribs,” ASME J. Turbomachinery, vol. 113, pp. 590-596, 1990.

[3] D. E. Metzger, R. A. Berry, and J. P. Bronson, "Developing Heat Transfer in Rectangular Ducts with Staggered Arrays of Short Pin Fins," ASME Journal of Heat Transfer, vol. 104, pp. 700-706, 1982.

[4] H. Xu, "Developing LES/DNS Simulation Capability based on Immersed Boundary Method coupled with FCAC Multigrid and AMR Techniques," in Proceedings of The $18^{\text {th }}$ International Conference on Finite Elements in Flow Problem, Taipei, 2015.

[5] H. Xu, M. Khalid and A. Pollard, "Large Eddy Simulation of Turbulent Flow in a Confined Square Coaxial Jet," International Journal of Computational Fluid Dynamics, vol. 17, no. 5, pp. 339-356, 2003.

[6] J. Kim, P. Moin, "Application of a fractional-step method to incompressible Navier-Stokes equations," Journal of Computational Physics, vol. 59, pp. 308-323, 1985.

[7] H. Xu, W. Yuan, M. Khalid, Design of a high-performance unsteady Navier-Stokes solver using flexible-cycle additive-correction multi-grid technique. Journal of Computational Physics, 209: 504-540, 2005. 